\title{
Invasive fungal diseases in adolescents and young adults after allogeneic hematopoietic stem cell transplantation
}

\author{
Oksana Ayzsilnieks', Marina Popova ${ }^{1}$, Alisa Volkova ${ }^{1}$, Olga Pinegina ${ }^{1}$, Svetlana Ignatyeva ${ }^{2}$, Kirill Ekushev ${ }^{1}$, Olga Sle- \\ sarchuk $^{1}$, Maria Vladovskaya ${ }^{1}$, Ludmila Zubarovskaya ${ }^{1}$, Nikolay Klimko ${ }^{1,2}$, Boris Afanasyev ${ }^{1}$ \\ ${ }^{1}$ R.Gorbacheva Memorial Research Institute of Children Oncology, Hematology and Transplantation, First St.Petersburg State \\ I.Pavlov Medical University, St.Petersburg, Russia; \\ ${ }^{2}$ Dept. of Clinical Mycology, I. Mechnikov North-Western State Medical University, St.Petersburg, Russia
}

Contact: Dr. Marina Popova

E-mail: marina.popova.spb@gmail.com

\section{Background}

Invasive fungal diseases (IFD) after allogeneic hematopoietic stem cell transplantation (allo-HSCT) are still actual problem despite improving prognosis due to recent introduction of novel antifungals and diagnostic procedures. There exist only limited data on IFD in adolescents and young adults (15 to 25 y.o.) after allo-HSCT. The aim of this study focuses on clinical characteristics, risk factors and outcomes of IFD in adolescents and young adults after allo-HSCT.

\section{Patients and Methods}

121 patients after first allo-HSCT were included into a retrospective single-center study from Jan 2013 to Nov 2015 (Table 1). Their age was from 15 to 25 years old, a median of 20 years. Underlying diseases were as follows: high risk acute leukemia, 74.4\%; lymphoma, $11.6 \%$; nonmalignant diseases, $7.4 \%$, others, $6.6 \%$. Allo-HSCT from matched unrelated donors was performed in $62.8 \%$, matched related donors, $24.8 \%$, and haploidentical grafting, $12.4 \%$. Reduced-intensity conditioning was used in $75.2 \%$ of the cases. EORTC/MSG 2008 criteria for IFD diagnosis were used, only probable and proven IFD were taken into analysis. Active diagnostic strategy, including bronchoscopy, was used before allo-HSCT, with bronchoalveolar lavage sampling in patients with CT scanning of the lung lesions. "Active IFD" was defined as IFD diagnosed just before allo-HSCT. IFD-events means a newly arising IFD, and relapse of IFD in those patients who had it before allo-HSCT.

\section{Results}

IFD was diagnosed before allo-HSCT in $19.8 \%$ of total case number (24/121): invasive aspergillosis (IA), in 15 patients, hepatosplenic candidiasis (HSC), in 5 cases, and one patient had four IFD (IA+HSC, IA + Mucormycosis, IA + PCP). Complete response to antifungal therapy was in 9 patients (37.5\%), partial response, in 6 subjects (25\%), and "active IFD", in 9 patients $(37.5 \%)$. For a half of cases with active IFD (41.7\%), a secondary antifungal prophylaxis was performed with Voriconazole. Patients free of IFD before allo-HSCT $(n=85)$ received primary prophylaxis with Fluconazole (70.2\%) or with Voriconazole/Posaconazole (5.8\%). Cumulative incidence of IFD events by two years after allo-HSCT was $15.7 \%$ (19/121), including 18 cases of new IFD, and one IA relapse. Median time to the onset of IFD event was D+43 (14 to 577). Incidence of IA was $8.4 \%(10 / 121)$, with median time to onset D+27.5 (14 to 577). Invasive candidiasis (IC) was documented for $1.1 \%$ of total group, with median time to onset D+226.5 (54 to 399); Pneumocystis pneumonia (PCP), $0.5 \%$ (since day+43). The main sites of infection were lungs (86.7\%). Risk factors for IFD development after allo-HSCT in the patients without pre-transplant IFD were: age $<18$ y.o. $(\mathrm{p}=0.03)$; non-malignant underlying disease $(\mathrm{p}=0.016)$, and acute GvHD $(\mathrm{p}=0.036)$. Presence of IFD before allo-HSCT $(\mathrm{p}=0.67)$, grade 4 neutropenia for more than 20 days ( $\mathrm{p}=0.08$ ), active underlying disease at the time of allo-HSCT $(\mathrm{p}=0.38)$, reactivation of CMV infection after allo-HSCT ( $\mathrm{p}=0.058)$, relapse of underlying disease within 100 days after allo-HSCT $(\mathrm{p}=0.65)$, and presence of chronic GvHD ( $p=0.19$ ) were not significantly associated with IFD development after allo-HSCT. Ten patients with IA $(41.7 \%)$ were treated with Voriconazole as a first line therapy, all the subjects with IC, with echinocandins, and a patient with PCP was treated with Trimethoprim/Sulfamethoxazole. A 12-week overall survival (OS) from the day of IFD diagnosis post-HSCT was $68.4 \%$ (Fig.1). The 12 -week OS in cases of IA was $62.5 \%$, and the patients with IC and PCP were alive at 12 weeks after IFD diagnosis. Two-year OS rates after allo-HSCT was $70.2 \%$. There was no statistical difference in the two-year OS for the cases with (66.7\%) or without IFD (71.1\%) detected before allo-HSCT $(\mathrm{p}=0.91)$. 


\section{Conclusions}

Incidence of IFD in adolescents and young adults before allo-HSCT was $19.8 \%$. Cumulative incidence of IFD at 2 years after allo-HSCT was $15.7 \%$. The main type of IFD was invasive aspergillosis (62.5\%). Only one patient with IA before allo-HSCT had a relapse of mycosis (5.3\%). Risk factors for IFD after allo-HSCT $(\mathrm{p}<0.05)$ were: age $<18$ y.o., non-malignant underlying disease, and acute GvHD. 12-week OS value after the post-transplant IFD diagnosis was $68.4 \%$. Due to effective diagnosis, timely treatment and secondary prophylaxis, the invasive fungal diseases, either before or after allo-HSCT, did not impair the outcome of HSCT in adolescents and young adults.

\section{Keywords}

Invasive fungal disease, allo-HSCT, adolescents and young adults, aspergillosis, pre-transplant mycosis, overall survival.

\section{Инвазивные микозы у реципиентов аллогенной трансплантации гемопоэтических стволовых клеток подросткового возраста и молодых взрослых}

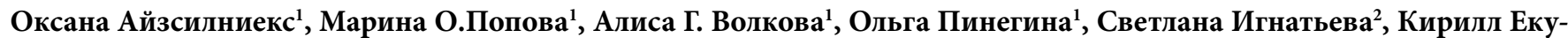
шев ${ }^{1}$, Ольга Слесарчук ${ }^{1}$, Мария Д. Владовская ${ }^{1}$, Людмила С. Зубаровская ${ }^{1}$, Николай Н. Климко ${ }^{1,2}$, Борис В. Афанасьев $^{1}$

${ }^{1}$ Первый Санкт-Петербургский государственный медицинский университет им. И.П. Павлова, НИИ Детской онкологии, гематологии и трансплантологии им. Р.М. Горбачевой, Санкт-Петербург.

${ }^{2}$ Кафедра клинической микологии, аллергологии, иммунологии, Северо-Западный Медицинский Университет им. И.И. Мечникова, Санкт-Петербург.

\section{Введение}

Несмотря на внедрение современных методов диагностики, профилактики и лечения, инвазивные микозы (ИМ) остаются актуальной проблемой после аллогенной трансплантации гемопоэтических стволовых клеток (алло-ТГСК). Целью настоящего исследования явилась оценка распространенности, этиологии, факторов риска развития ИМ и общей выживаемости $(\mathrm{OB})$ у реципиентов алло-ТГСК подросткового возраста и молодых взрослых.

\section{Материалы и методы}

Ретроспективно анализировались данные 121 пациента в возрасте от 15 до 25 лет, которым была выполнена первая алло-ТГСК в период с января 2013 г. по ноябрь 2015 г. в клинике Института детской онкологии, гематологии и трансплантологии им. Р.М. Горбачевой ПСПбГМУ им. акад. И.П. Павлова (Табл. 1). Диагноз ИМ установлен согласно критериям EORTC/MSG 2008. «активный» ИМ - диагноз микоза установлен непосредственно перед ТГСК.

Table 1. Clinical characteristics of the patients with invasive fungal disease

\begin{tabular}{|l|l|l|l|}
\hline Variable & $\mathrm{N}=121(\%)$ & Variable & $\mathrm{N}=121(\%)$ \\
\hline Age & 20 (median: 15-25) & $\begin{array}{l}\text { HLA-matching } \\
\text { Matched }\end{array}$ & $89(73,6)$ \\
\hline $\begin{array}{l}\text { Diagnosis } \\
\text { Non-malignant disease }\end{array}$ & $53(43,8)$ & Mismatched & $32(26,4)$ \\
\hline Acute lymphocytic leucemia & $53(43,8)$ & $\begin{array}{l}\text { Posttransplant period } \\
4^{\text {th }} \text { grade neutropenia }\end{array}$ & $22(18,20$ \\
\hline Acute myeloid leucemia & $37(30,6)$ & Acute GvHD (I-IV) & $51(42,1)$ \\
\hline Lymphoma & $14(11,6)$ & Relapsw of underlying disease & $15(12,4)$ \\
\hline Status of underlying disease & $81(66,9)$ & Chronic GvHD & $33(27,3)$ \\
\hline
\end{tabular}




\begin{tabular}{|l|l|l|l|}
\hline Variable & $\mathrm{N}=121(\%)$ & Variable & $\mathrm{N}=121(\%)$ \\
\hline Active disease & $40(33,1)$ & $\begin{array}{l}\text { Antifungal prophilaxis } \\
\text { Primary with fluconazole }\end{array}$ & $85(70,2)$ \\
\hline Myeloablative congitioning & $30(24,8)$ & Secondary with echinocandins & $6(4,9)$ \\
\hline Reduced intensity conditioning & $91(75,2)$ & Secondary with voriconazole & $10(8,3)$ \\
\hline $\begin{array}{l}\text { GvHD prophylaxis } \\
\text { ATG }\end{array}$ & $26(21,5)$ & $\begin{array}{l}\text { Primary with voriconazole/ } \\
\text { posaconazole }\end{array}$ & $7(5,8)$ \\
\hline Cyclophosphan & $95(78,5)$ & IFD before allo-HSCT & $24(19,8)$ \\
\hline $\begin{array}{l}\text { Type of HSCT } \\
\text { Matched, unrelated }\end{array}$ & $76(62,8)$ & Invasive aspergillosis & $15(16,5)$ \\
\hline Matched, related & $30(24,8)$ & Invasive candidiasis & $5(20,8)$ \\
\hline Haplo & $15(12,4)$ & $\begin{array}{l}\text { IA+IC/IA+Mucormycosis/ } \\
\text { IA+PCP }\end{array}$ & $4(16,7)$ \\
\hline
\end{tabular}

2-year OS after allo-HSCT

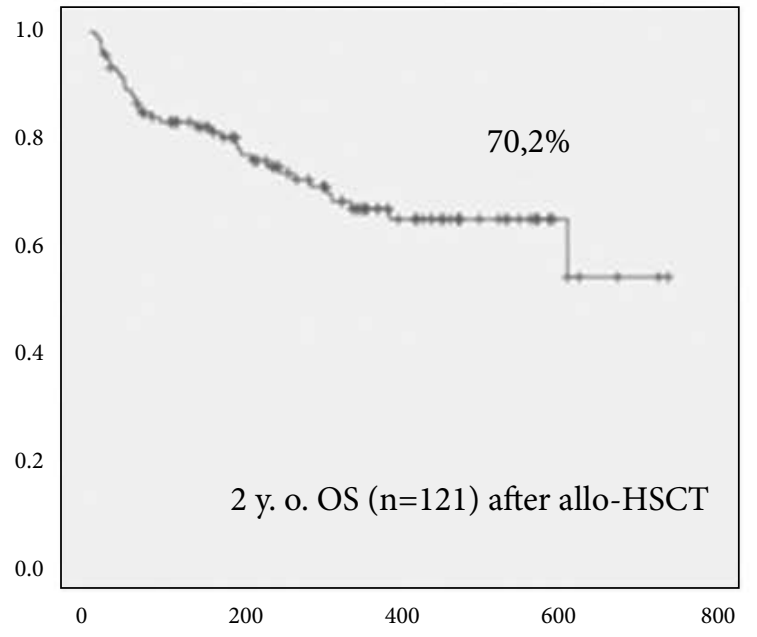

days after allo-HSCT

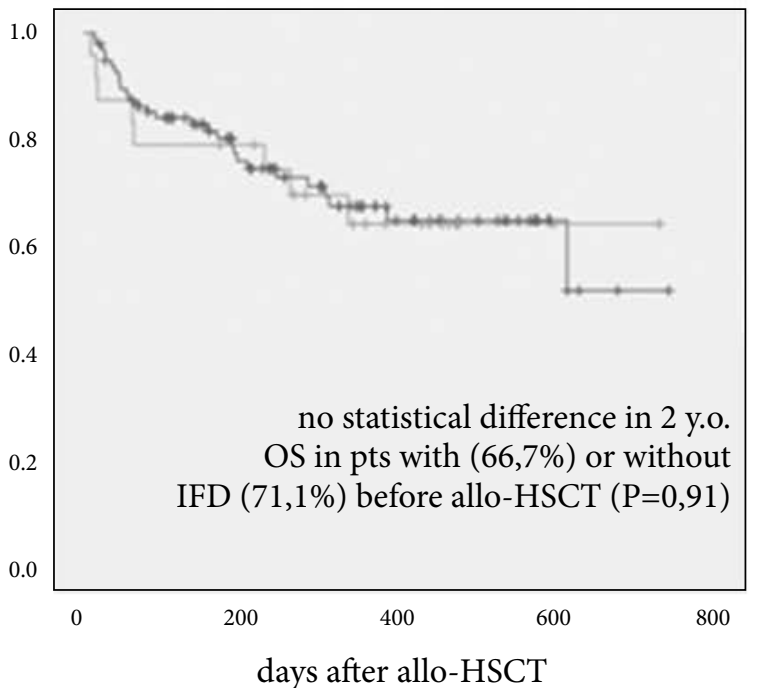

days after allo-HSCT

Fig.1. Overall survival rates in the patients with vs without invasive fungal disease

\section{Результаты}

Частота ИМ до алло-ТГСК - 19,8\% (n=24): инвазивный аспергиллез (ИА) $\mathrm{n}=15$, инвазивный кандидоз (ИК) $\mathrm{n}=5$, и у 4-х пациентов было два ИМ (ИА+ИК, ИА+мукормикоз, пневмоцистная пневмония+ИА). Полный ответ на противогрибковую терапию был у $37,5 \%$, частичный ответ - 25\%, «активный» ИМ до ТГСК у $37,5 \%$. Больным с имевшимся до алло-ТГСК ИМ и полным и/или частичным ответом на противогрибковую терапию проводили вторичную антифунгальную профилактику вориконазолом (41,7\%). Остальные пациенты $(\mathrm{n}=85)$ получали первичную антифунгальную профилактику преимущественно флуконазолом (70,2\%), или вориконазолом/позаконазолом - 5,8\%. Кумулятивная частота ИМ после алло-ТГСК в течение 2-х лет - 15,7\%, включая новый ИМ $(\mathrm{n}=18)$ и рецидив ИА $(\mathrm{n}=1)$ с медианой дня возникновения Д+43 (14-577). Факторы риска развития ИМ после алло-ТГСК: возраст $<18$ лет $(\mathrm{p}=$ $0,03)$, незлокачественные заболевания крови $(\mathrm{p}=0,016)$, острая РТПХ $(\mathrm{p}=0,036)$. Наличие ИМ до алло-ТГСК $(\mathrm{p}=0,67)$, нейтропения 4 степени более 20 дней $(\mathrm{p}=0,08)$, рецидив основного заболевания на момент алло-ТГСК $(\mathrm{p}=0,38)$, реактивация ЦМВ инфекции после алло-ТГСК $(\mathrm{p}=0,058)$, рецидив основного заболевания до 100 дня после алло-ТГСК $(\mathrm{p}=0,65)$ и наличие хронической РТПХ $(\mathrm{p}=0,19)$ не влияли на развитие ИМ после ТКМ. Десять пациентов $(41,7 \%)$ с ИА получали лечение вориконазолом в качестве первой линии терапии, все пациенты с ИК - эхинокандины, пациент с пневмоцистной пневмонией - триметоприм-сульфаметоксазол. ОВ пациентов в течение 12 недель от диагностики ИМ составила 68,4\%, ИА - 62,5\%, пациенты с ИК и пневмоцистной пневмонией живы (Рис.1). Общая 2-х летная выживаемость у пациентов с наличием ИМ до алло-ТГСК $(66,7 \%)$ не отличалась от выживаемости пациентов без ИМ (71,1\%, $\mathrm{p}=0.91)$. 


\section{Выводы}

Частота ИМ у реципиентов алло-ТГСК подросткового возраста и молодых взрослых до алло-ТГСК составила 19,8\%. Кумулятивная частота ИМ после алло-ТГСК (2 года) - 15,7\%. Основной возбудитель - Aspergillus spp. (62,5\%). Только один пациент с ИА имел рецидив $(5,3 \%)$ после ТКМ. Факторами риска развития ИМ после алло-ТГСК являлись $(\mathrm{p}<0,05):$ возраст $<18$ лет, незлокачественное заболевание крови, острая РТПХ. 12-недель- ная выживаемость с момента диагностики ИМ после ТГСК составила $68,4 \%$. Наличие ИМ до и после ТКМ не влияло на исход трансплантации у данной возрастной группы пациентов.

\section{Ключевые слова}

Инвазивные микозы, алло-ТГСК, подростки и молодые взрослые, аспергиллёз, микозы до трансплантации, общая выживаемость. 